POS $\quad \begin{aligned} & \text { PROCEEDINGS } \\ & \text { OF SCIENCE }\end{aligned}$

\title{
Hadronic Charm Decays
}

\section{Jonas Rademacker*广}

$H$ H Wills Physics Laboratory, University of Bristol, UK

E-mail: Jonas.Rademacker@bristol.ac.uk

Flavor Physics and CP Violation - FPCP 2010

May 25-29, 2010

Turin, Italy

* Speaker.

$\dagger$ on behalf of the CLEO-c collaboration. 


\section{Introduction}

Hadronic charm decays provide insights into both electroweak and strong dynamics. This includes the study of long-distance hadronic effects, the approximate symmetries of strong interactions, and precision tests of the Standard Model. In these proceedings we summarise recent results of branching fraction measurements of $\mathrm{D}^{0}, \mathrm{D}^{ \pm}$, and $\mathrm{D}_{\mathrm{s}}^{ \pm}$mesons, including measurements of relative and absolute branching fractions in inclusive and exclusive modes, exploring flavour symmetries, strong phases, and Dalitz analyses. Other aspects of hadronic charm decays are covered elsewhere in these proceedings $[1,2,3,4,5]$.

\section{Charm decays to two pseudoscalars}

CLEO-c has recently published the results of branching fractions of $\mathrm{D}^{0}, \mathrm{D}^{+}$, and $\mathrm{D}_{\mathrm{s}}$ decays to two pseudoscalars, based on an analysis of CLEO-c's full data set [6], with $818 \mathrm{pb}^{-1}$ at $\psi(3770)$ corresponding to $3 \cdot 10^{6} \mathrm{D}^{0} \overline{\mathrm{D}}^{0}$ pairs and $2.4 \cdot 10^{6} \mathrm{D}^{+} \mathrm{D}^{-}$pairs; and $586 \mathrm{pb}^{-1}$ at $\sqrt{s}=4170 \mathrm{MeV}$ corresponding to $5.3 \cdot 10^{5} \mathrm{D}_{\mathrm{s}}^{ \pm} \mathrm{D}_{\mathrm{s}}^{\mp *}$ pairs. Many of the resulting branching fraction measurements are more precise than the previous world average [8], and some decay modes have been seen for the first time. Bhattacharya \& Rosner [7] have analysed these results in terms of the diagrammatic approach $[9,10,11,12]$. The decay amplitudes are expressed in terms of topological quark-flow diagrams; the diagrams used in this analysis are given in Fig. 1. Flavour symmetries of the strong interaction are used to express different $\mathrm{D}^{0}, \mathrm{D}^{ \pm}$and $\mathrm{D}_{\mathrm{s}}^{ \pm}$two-body decay amplitudes in terms of the same set of six diagrams. These results are then used to predict the decay amplitudes of singly Cabibbo suppressed (SCS) and doubly Cabibbo suppressed (DCS) two body decays by assuming that the SCS (DCS) amplitudes are the CF amplitudes, scaled by a factor $\lambda=\sin \theta_{c}\left(\lambda^{2}=\sin ^{2} \theta_{c}\right)$ where $\theta_{c}$ is the Cabibbo angle. The predictions for decays involving kaons and pions, only, are mostly in reasonable agreement with measurement although the approach considerably overestimates $\mathscr{B}\left(\mathrm{D}^{0} \rightarrow \pi^{+} \pi^{-}\right)$and underestimates $\mathscr{B}\left(\mathrm{D}^{0} \rightarrow \mathrm{K}^{+} \mathrm{K}^{-}\right)$. For SCS decays involving $\eta$ and $\eta^{\prime}$, there are indications for a non-negligible contribution from the singlet annihilation (SE) diagram. For a comprehensive review of hadronic charm decays and their analysis using this and other methods see also [13].
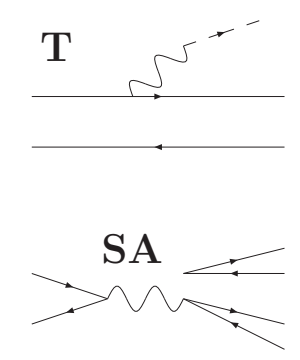
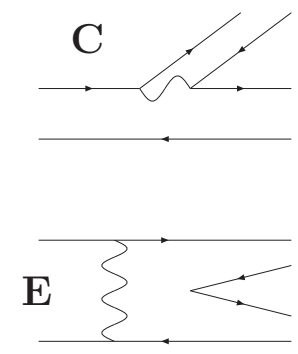
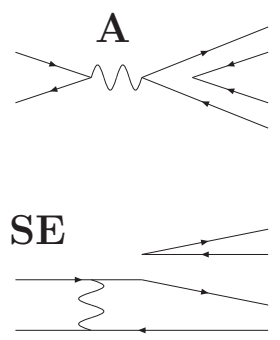

Figure 1: Quark flow diagrams used in the analysis of CLEO-c's D $\rightarrow$ PP data [6] by Bhattacharya \& Rosner [7]: Tree, Colour-suppressed tree, Annihiliation, Singlet-emission with Annihilation, Exchange, and Singlet-emission with Exchange. 


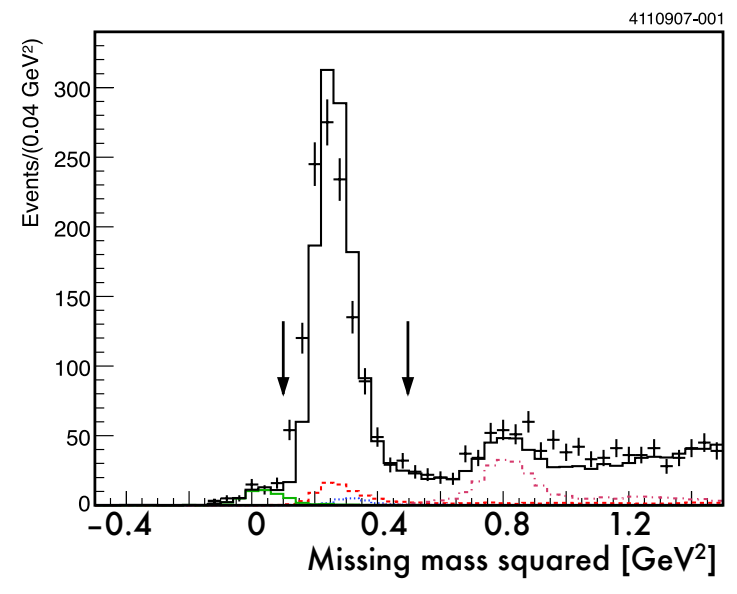

(a) $\mathrm{D}^{0} \rightarrow \mathrm{K}_{\mathrm{L}} \pi^{0}$

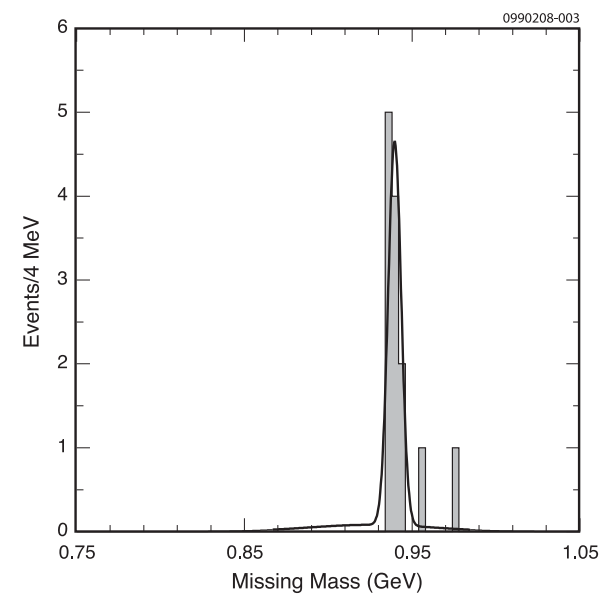

(b) $\mathrm{D}_{\mathrm{s}}^{+} \rightarrow \mathrm{p} \overline{\mathrm{n}}$

Figure 2: Missing mass distributions at CLEO-c. Figure (a) shows the missing mass-squared in the reconstruction of $\mathrm{D}^{0} \rightarrow \mathrm{K}_{\mathrm{L}} \pi^{0}$ at CLEO-c [17]. The points with error bars represent the data; the solid line Monte Carlo simulation; and dashed, colored lines simulations of the peaking backgrounds. The slight shift in the peak position is understood and due to a minor discrepancy in the calorimeter simulation at large photon energies. Figure (b) shows the missing mass of the $n$ in $\mathrm{D}_{\mathrm{s}}^{+} \rightarrow \mathrm{p} \bar{n}$ decays CLEO-c [18].

\section{3. $\mathrm{K}^{0}, \overline{\mathrm{K}}^{0}$ interference}

As pointed out by Bigi \& Yamamoto [14], the decay rates of $\mathrm{D}^{0} \rightarrow \mathrm{K}_{\mathrm{S}} \pi^{0}$ and $\mathrm{D}^{0} \rightarrow \mathrm{K}_{\mathrm{L}} \pi^{0}$ are not the same because of the interference of the CF component $\mathrm{D}^{0} \rightarrow \overline{\mathrm{K}}^{0} \pi^{0}$ with the DCS $\mathrm{D}^{0} \rightarrow \mathrm{K}^{0} \pi^{0}$ component which enters with a different sign for decays to $\mathrm{K}_{\mathrm{L}}$ and $\mathrm{K}_{\mathrm{S}}$ :

$$
A\left(\mathrm{D}^{0} \rightarrow \mathrm{K}_{\mathrm{S}, \mathrm{L}} \pi^{0}\right)=A\left(\mathrm{D}^{0} \rightarrow \overline{\mathrm{K}}^{0} \pi^{0}\right) \pm A\left(\mathrm{D}^{0} \rightarrow \mathrm{K}^{0} \pi^{0}\right)
$$

Assuming U-spin symmetry of the strong interaction, the decay rate asymmetry is given by [14]:

$$
A_{K_{S, L} \pi^{0}}=\frac{\Gamma\left(\mathrm{D}^{0} \rightarrow K_{S} \pi^{0}\right)-\Gamma\left(\mathrm{D}^{0} \rightarrow K_{L} \pi^{0}\right)}{\Gamma\left(\mathrm{D}^{0} \rightarrow K_{S} \pi^{0}\right)+\Gamma\left(\mathrm{D}^{0} \rightarrow K_{L} \pi^{0}\right)}=2 \tan ^{2} \theta_{c}=0.109
$$

A measurement of $A_{K_{S, L} \pi^{0}}$ therefore provides a test of U-spin symmetry, which is important for example for extracting the CP-violating parameter $\gamma$ from $\mathrm{B}_{\mathrm{s}} \rightarrow \mathrm{KK}$ and $\mathrm{B}_{\mathrm{d}} \rightarrow \pi \pi$ decays $[15,16]$. The reconstruction of $\mathrm{D}^{0} \rightarrow \mathrm{K}_{\mathrm{L}} \pi^{0}$ is challenging because it involves two neutral particles. CLEO-c uses its CsI calorimeter to identify the $\pi^{0}$. The four-momentum of the practically invisible $\mathrm{K}_{\mathrm{L}}$ is reconstructed using beam constraints, benefiting from the very clean environment at CLEO-c where the DD̄ pairs produced absorb the entire beam energy. The resulting missing mass-squared distribution is shown in Fig. 2(a). The asymmetry, measured in $281 \mathrm{pb}^{-1}$ of data, is [17]:

$$
A_{K_{S, L} \pi^{0}}=0.108 \pm 0.025 \pm 0.024
$$

which is in excellent agreement with the prediction by [14] based in U-spin symmetry. Theoretical prediction for the related asymmetry

$$
A_{K_{S, L} \pi^{+}}=\frac{\Gamma\left(\mathrm{D}^{+} \rightarrow K_{S} \pi^{+}\right)-\Gamma\left(\mathrm{D}^{+} \rightarrow K_{L} \pi^{+}\right)}{\Gamma\left(\mathrm{D}^{+} \rightarrow K_{S} \pi^{+}\right)+\Gamma\left(\mathrm{D}^{+} \rightarrow K_{L} \pi^{+}\right)}
$$


are more difficult as there is no such clean symmetry. Using SU(3), Gao predicts [19] $A_{K_{S, L} \pi^{+}} \approx$ 0.04. Based on the diagrammatic approach, Bhattacharya \& Rosner [7] predict $A_{K_{S, L} \pi^{+}}=-0.005 \pm$ 0.013. Both are consistent with CLEO-c's measurement [17] of $A_{K_{S, L} \pi^{+}}=0.022 \pm 0.016 \pm 0.018$.

\section{4. $\mathrm{D} \rightarrow \mathrm{KK} \pi, \mathrm{D} \rightarrow \mathrm{K} \pi \pi$}

These decay modes allow a test of $S U(3)_{F}$ through the double ratio [20]

$$
\frac{\Gamma\left(\mathrm{D}^{+} \rightarrow \mathrm{K}^{+} \mathrm{K}^{+} \pi^{-}\right)}{\Gamma\left(\mathrm{D}^{+} \rightarrow \mathrm{K}^{+} \mathrm{K}^{-} \pi^{+}\right)} \frac{\Gamma\left(\mathrm{D}^{+} \rightarrow \mathrm{K}^{+} \pi^{+} \pi^{-}\right)}{\Gamma\left(\mathrm{D}^{+} \rightarrow \mathrm{K}^{-} \pi^{+} \pi^{+}\right)}=\tan ^{8} \theta
$$

where $\theta_{c}$ is the Cabibbo angle. The BELLE collaboration find $(1.57 \pm 0.21) \times \tan ^{8} \theta_{C}$ [21], which is neither in particularly good agreement or disagreement with the $S U(3)_{F}$ assumption. In the same paper, BELLE report the first observation of $\mathrm{D}_{\mathrm{s}}^{+} \rightarrow \mathrm{K}^{+} \mathrm{K}^{+} \pi^{-}$.

5. $\mathrm{D}^{0} \rightarrow \mathrm{p} \overline{\mathrm{n}}$

The first observation of a meson decaying to two baryons has been made by CLEO-c in the mode $\mathrm{D}_{\mathrm{s}}^{+} \rightarrow \mathrm{p} \overline{\mathrm{n}}$, which is also the only kinematically allowed baryonic decay of a light charm meson $\left(\mathrm{D}^{0}, \mathrm{D}^{+}\right.$, or $\left.\mathrm{D}_{\mathrm{s}}\right)$. CLEO-c reconstruct the anti-neutron from the missing mass with virtually no background, as shown in Fig. 2(b). CLEO-c measures the following branching fraction [18]:

$$
\mathscr{B}\left(\mathrm{D}_{\mathrm{s}}^{+} \rightarrow p \bar{n}\right)=\left(1.30 \pm 0.36_{-0.16}^{+0.12}\right) \cdot 10^{-3}
$$

This decay mode is dominated by long-distance effects. Chen, Cheng and Hsio [22] estimate these as $\mathscr{B}\left(\mathrm{D}_{\mathrm{s}}^{+} \rightarrow p \bar{n}\right) \approx\left(0.8_{-0.6}^{+2.4}\right) \cdot 10^{-3}$ in agreement with CLEO-c's observation - short-distance contributions from the annihilation diagram are about 3 orders of magnitude smaller.

\section{Absolute Branching Fractions}

Absolute Branching fraction measurements are particularly important for those decays frequently used as normalisation modes. BaBar, BELLE and CLEO-c published measurements of absolute branching fractions, using different techniques: BaBar obtains a normalisation by reconstructing $\mathrm{D}^{*} \rightarrow \mathrm{D} \pi$ using only the slow pion in this decay chain, and information from the rest of the event, but not the D itself [23]. CLEO-c produces charm mesons always in pairs, either $\mathrm{e}^{+} \mathrm{e}^{-} \rightarrow \psi(3770) \rightarrow \mathrm{DD}$ for $\mathrm{D}^{0}$ or $\mathrm{D}^{ \pm}$, or $\mathrm{e}^{+} \mathrm{e}^{-} \rightarrow \mathrm{D}^{ \pm} \mathrm{D}^{* \mp}$. One charm meson provides the normalisation for the decay rates of the other $[24,25]$. BELLE uses the process $\mathrm{e}^{+} \mathrm{e}^{-} \rightarrow \mathrm{D}_{\mathrm{s}}^{*+} \mathrm{D}_{\mathrm{s} 1}^{-}$with $\mathrm{D}_{\mathrm{s} 1}^{-} \rightarrow \overline{\mathrm{D}}^{* 0} \mathrm{~K}^{-}$, one charm meson provides the normalisation for the other [26]. A frequently-used normalisation mode for $D_{s}$ branching ratios is the decay $D_{s} \rightarrow \phi \pi$. This, however, is problematic because of interference effects in the $\mathrm{K}^{+} \mathrm{K}^{-} \pi^{+}$Dalitz plot, in particular from $\mathrm{f}(980)[28,29,30]$. CLEO-c therefore publishes the absolute branching fraction for $\mathrm{D}_{\mathrm{s}}^{+} \rightarrow \mathrm{K}^{+} \mathrm{K}^{-} \pi^{+}$, including the entire phase space. However, when using this as a normalisation mode, it can be advantageous to select events with a $\mathrm{K}^{+} \mathrm{K}^{-}$invariant mass near the $\phi$ mass, in order to reject background. To accommodate this, CLEO-c also publishes branching fractions for parts of the $\mathrm{D}_{\mathrm{s}}^{+} \rightarrow \mathrm{K}^{+} \mathrm{K}^{-} \pi^{+}$ phase space corresponding to different cuts around the $\phi$ mass, but without making any statement about the contribution of $\mathrm{D}_{\mathrm{s}}^{+} \rightarrow \phi \pi$ this includes. The absolute $\mathrm{D}_{\mathrm{s}}$ branching fractions for different decay modes from this analysis [25] are given in Tab. 1. 
Table 1: Results from CLEO-c's recent measurement of absolute $D_{s}$ branching fractions [25], the world average branching fractions before CLEO-c's measurement [27], ratios of branching fractions to $\mathscr{B}\left(\mathrm{D}_{\mathrm{s}}^{+} \rightarrow\right.$ $K^{-} K^{+} \pi^{+}$), and charge asymmetries $\mathscr{A}_{C P}$. Uncertainties on CLEO-c measurements are statistical and systematic, respectively. Table reproduced from [25]

\begin{tabular}{lcccc}
\hline \hline \multirow{2}{*}{ Mode } & $\begin{array}{l}\text { CLEO-c result } \\
\mathscr{B}(\%)[25]\end{array}$ & $\begin{array}{c}\text { PDG 2007 fit } \\
\mathscr{B}(\%)[27]\end{array}$ & $\mathscr{B} / \mathscr{B}\left(K^{-} K^{+} \pi^{+}\right)$ & $\mathscr{A}_{C P}(\%)$ \\
\hline$K_{S}^{0} K^{+}$ & $1.49 \pm 0.07 \pm 0.05$ & $2.2 \pm 0.4$ & $0.270 \pm 0.009 \pm 0.008$ & $+4.9 \pm 2.1 \pm 0.9$ \\
$K^{-} K^{+} \pi^{+}$ & $5.50 \pm 0.23 \pm 0.16$ & $5.3 \pm 0.8$ & 1 & $+0.3 \pm 1.1 \pm 0.8$ \\
$K^{-} K^{+} \pi^{+} \pi^{0}$ & $5.65 \pm 0.29 \pm 0.40$ & - & $1.03 \pm 0.05 \pm 0.08$ & $-5.9 \pm 4.2 \pm 1.2$ \\
$K_{S}^{0} K^{-} \pi^{+} \pi^{+}$ & $1.64 \pm 0.10 \pm 0.07$ & $2.7 \pm 0.7$ & $0.298 \pm 0.014 \pm 0.011$ & $-0.7 \pm 3.6 \pm 1.1$ \\
$\pi^{+} \pi^{+} \pi^{-}$ & $1.11 \pm 0.07 \pm 0.04$ & $1.24 \pm 0.20$ & $0.202 \pm 0.011 \pm 0.009$ & $+2.0 \pm 4.6 \pm 0.7$ \\
$\pi^{+} \eta$ & $1.58 \pm 0.11 \pm 0.18$ & $2.16 \pm 0.30$ & $0.288 \pm 0.018 \pm 0.033$ & $-8.2 \pm 5.2 \pm 0.8$ \\
$\pi^{+} \eta^{\prime}$ & $3.77 \pm 0.25 \pm 0.30$ & $4.8 \pm 0.6$ & $0.69 \pm 0.04 \pm 0.06$ & $-5.5 \pm 3.7 \pm 1.2$ \\
$K^{+} \pi^{+} \pi^{-}$ & $0.69 \pm 0.05 \pm 0.03$ & $0.67 \pm 0.13$ & $0.125 \pm 0.009 \pm 0.005$ & $+11.2 \pm 7.0 \pm 0.9$ \\
\hline \hline
\end{tabular}

\section{Inclusive $D_{s} B F$ and exclusive $D_{s} \rightarrow \omega X$}

In 2009, CLEO-c published a measurement the inclusive branching fractions of $D_{s}$ in modes [31], such as $\mathrm{D}_{\mathrm{s}}^{+} \rightarrow \pi^{+} \mathrm{X}, \mathrm{D}_{\mathrm{s}}^{+} \rightarrow \pi \mathrm{X}$, etc, where $\mathrm{X}$ stands for any combination of particles. While most inclusive branching fractions measured are compatible with the sum of known exclusive rates [32], this was initially not the case for the inclusive branching fraction $\mathscr{B}\left(\mathrm{D}_{\mathrm{s}} \rightarrow \omega \mathrm{X}\right)$, where $X$ stands for any combination of particles. CLEO-c measures this to be $(6.1 \pm 1.4) \%$, far more than the only known exclusive $\omega$ mode at the time, $\mathscr{B}\left(\mathrm{D}_{\mathrm{s}} \rightarrow \pi^{+} \omega\right)=(0.25 \pm 0.09) \%$ [8]. Since then, CLEO-c has searched for the missing exclusive decay modes to $\omega$, and found them [33]. The missing exclusive modes are mainly those were $X=\pi^{+} \pi^{0}(\mathscr{B}=(2.78 \pm 0.65 \pm 0.25) \%)$ and $X=\pi^{+} \pi^{-} \pi^{-}$ $(\mathscr{B}=(1.58 \pm 0.45 \pm 0.09) \%)$. The full results are given in [33].

\section{Dalitz Plots}

The kinematics of a 3 body decay $\mathrm{D} \rightarrow \mathrm{A}, \mathrm{B}, \mathrm{C}$ ( such as $\mathrm{D}^{+} \rightarrow \mathrm{K}^{+} \mathrm{K}^{-} \pi^{+}$) can be fully described by 2 parameters. In terms of the four momenta of the three decay products, which we will denote as $p_{A}, p_{B}, p_{C}$, one usually picks the following invariant-mass-squared parameters: $m_{A B}^{2} \equiv$ $\left(p_{A}+p_{B}\right)^{2}, \quad m_{B C}^{2} \equiv\left(p_{B}+p_{C}\right)^{2}$. A Dalitz plot [34] is the decay rate in terms of these or equivalent variables, displayed in a 2-dimensional plot. The full decay rate is given by [35]:

$$
\frac{d^{2} \Gamma}{d\left(m_{A B}^{2}\right) d\left(m_{B C}^{2}\right)}=\left|a_{1} e^{i \delta_{1}}+a_{2} e^{i \delta_{2}}+\ldots\right|^{2} \frac{\pi \sqrt{\lambda}}{2 m_{D}^{2}}
$$

with $\lambda=\left(m_{D}^{2}-m_{A}^{2}-m_{B}^{2}\right)^{2}-4 m_{A}^{2} m_{B}^{2}$ within the kinematically allowed limits, and $\lambda=0$ outside. In the above expression, $a_{i} e^{i \delta_{i}}$ describe complex contributions to the total decay amplitude. Herein lies the power of Dalitz analyses: the access not only to magnitudes, but also to phases. In the simplest case, $a_{i} e^{i \delta_{i}}$ are complex Breit-Wigner distributions (or similar e.g. the Flatté distribution [36]) describing individual particle resonances, with additional factors taking into account angular 

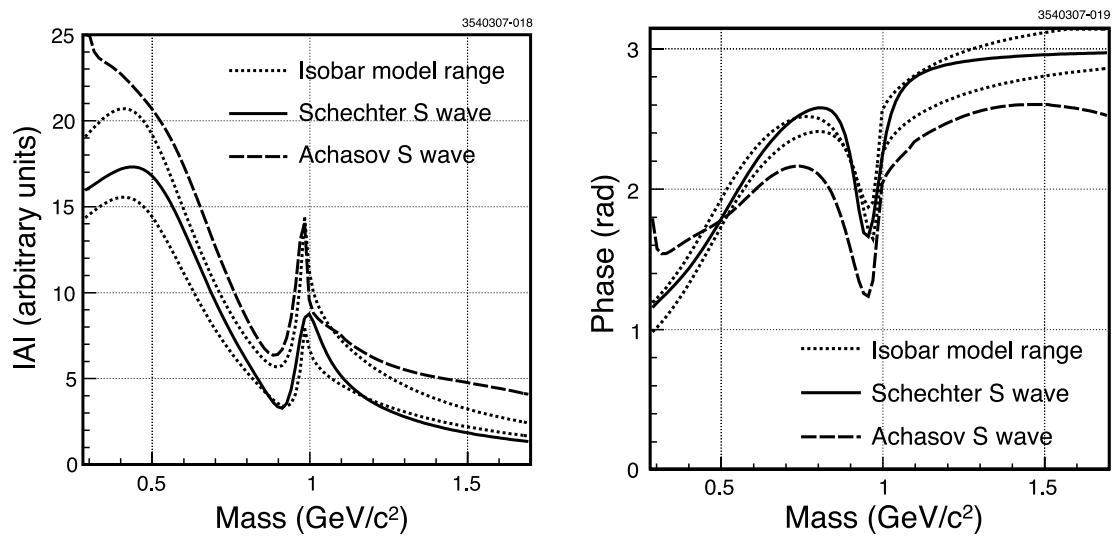

Figure 3: The magnitude and phase of the $S$ wave component for the different models considered in CLEOc's $\mathrm{D}^{0} \rightarrow \pi^{+} \pi^{-} \pi^{+}$analysis [42]. The dotted lines represent the upper and lower limit of the range of values obtained for the various isobar models. The broken line corresponds to Achasov's model, the solid line to Schechter's model. There is good agreement between the models.

momentum conservation, and form factors (Blatt-Weisskopf penetration factors [37]). This socalled isobar model has some shortcomings, the most severe one being that it violates unitarity, especially in the case of wide, overlapping resonances. More complicated models such as the K-matrix formalism [38, 39], which respects unitarity, may therefore be necessary to adequately describe the observed data, and to provide a theoretically satisfactory model. The general consensus - at least amongst experimentalists - appears to be that the isobar description is adequate for $P$ and $D$ wave resonances, but not for wide $S$ wave resonances. The adequate description of $L=0$ decays is the topic of the first three subsections below. Further we briefly discuss 4-body generalisations of Dalitz plots, and their use in mixing and CP violation measurements.

8.1 $\mathrm{D}_{\mathrm{s}}^{+} \rightarrow \mathrm{K}^{-} \mathrm{K}^{+} \pi^{+}$

In one of its most recent Dalitz analyses, based on its unique $\mathrm{D}_{\mathrm{s}}$ sample, CLEO-c published a Dalitz model analyses of the decay $\mathrm{D}_{\mathrm{s}}^{+} \rightarrow \mathrm{K}^{-} \mathrm{K}^{+} \pi^{+}$, based on $12 \mathrm{k}$ signal events [] The fit model is based on a previous isobar analysis by E687 with a far smaller sample (701 events) [] Compared to the original model, CLEO-c find a significant improvement in the fit quality through an additional $K K \mathrm{~S}$-wave component. The best fit is achieved with the further addition of a $f_{0}(1370)$ resonance.

8.2 $\mathrm{D}^{+} \rightarrow \pi^{+} \pi^{-} \pi^{+}$

Recent analyses of this channel include E791's analysis using an isobar fit with a $\sigma$ resonance [40], and FOCUS, who pioneered the K-matrix approach in this channel, and also analyse $\mathrm{D}_{\mathrm{S}} \rightarrow \pi^{+} \pi^{-} \pi^{+}$in their study [41]. The most recent result is by CLEO-c [42], using $\sim 2600$ signal events. CLEO consider isobar models with different descriptions of the $\mathrm{f}_{0}(980)$ and $\sigma$, and two models that respect unitarity and chirality, one according to Schechter [43] and another developed by Achasov [44]. All models considered agree with each other and the results are consistent with previous fits. The amplitude and phase of the $S$ wave contribution as function of $\pi^{+} \pi^{-}$invariant mass is reproduced in figure Fig. 3. 

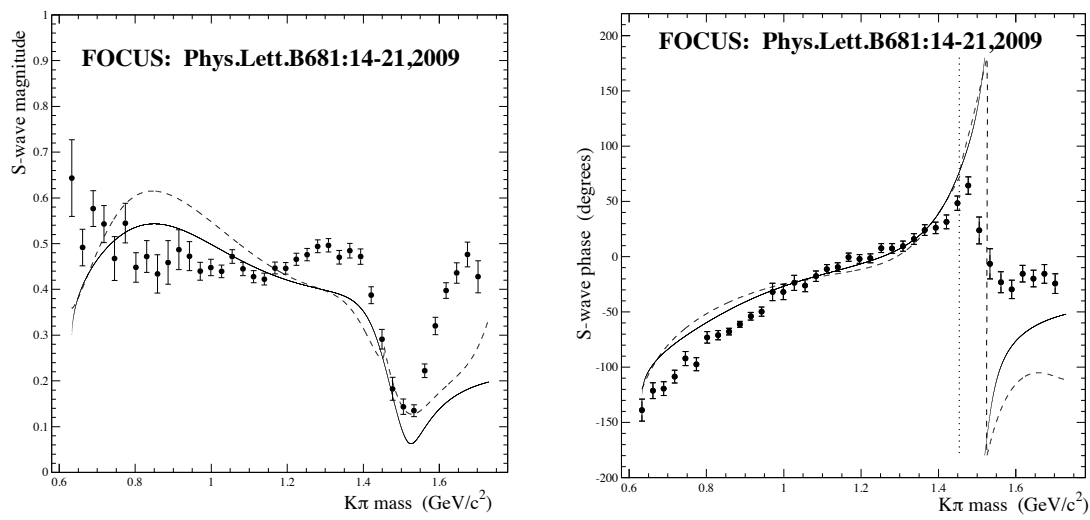

Figure 4: FOCUS model-independent analysis of the $\mathrm{K} \pi \mathrm{S}$-wave component in $\mathrm{D}^{+} \rightarrow \mathrm{K}^{-} \pi^{+} \pi^{-}$[50]. Points with error bars represent the result of the model-independent (binned) fit, the solid line the S-wave component of the isobar model and the broken line the S-wave component of the K-matrix model.

\section{3 $\mathrm{D}^{+} \rightarrow \mathrm{K}^{-} \pi^{+} \pi^{+}$}

The branching fraction of $\mathrm{D}^{+} \rightarrow \mathrm{K}^{-} \pi^{+} \pi^{+}$is comparably large, with $(9.51 \pm 0.34) \%$ [45]. Over $60 \%$ of its decay rate proceeds via a $\mathrm{K} \pi S$-wave, as has been observed in several experiments. In 2002 E791 [46], using an isobar model, found a large $\kappa$ contribution. In 2006, E791 re-analysed the same data with a model-independent description of the $S$ wave, using a binned amplitude and phase [47]. In 2007, FOCUS [48] applied the K-matrix formalism, constrained by LASS scattering data [49], to 54k events. Recently, FOCUS re-analysed the same data using a model-independent approach [50]. Figure 4 shows the magnitude and phase of the model independent S-wave component compared to the S-wave component of the previous isobar fit and a K-matrix fit, showing some interesting differences between the approaches [50]. The result based on the largest data set is from CLEO-c in 2008, using 140k events, with very little background (1.1\%) [51]. CLEO-c fit the data using both the isobar and the model-independent approach, and compare their result to models used by other experiments. For both types of model, CLEO-c get a significantly improved fit if they allow for an isospin $=2 \pi^{+} \pi^{-} S$ wave contribution, where the model-independent approach gives the better $\chi^{2}$ per degree of freedom.

\subsection{Four-body Amplitude Analyses}

Essentially the same formalism as for 3 body decays can be applied to to 4 body decay. Such analyses are challenging as the equivalent of the Dalitz plot now has 5 dimensions instead of 2 , phase space is not flat in the usual invariant-mass squared variables, and the amplitude structure is more complex. A recent example of such an analysis, using an isobar model, is given by FOCUS for the decay channel $\mathrm{D}^{0} \rightarrow \pi^{+} \pi^{+} \pi^{-} \pi^{-}$[52]. FOCUS observe that $\mathrm{D}^{0} \rightarrow \mathrm{a}_{1}(1260) \pi$ is the dominant decay channel, followed by $\mathrm{D}^{0} \rightarrow \rho \rho$. The authors find that the $\mathrm{a}_{1}$ predominantly decays to $\sigma \pi$. Many more results can be found in the paper, including the $\rho \rho$ polarisation.

\subsection{Mixing and CP violation}

Dalitz analyses are sensitive to phases and provide a precision tool for a variety of analyses in 
charm, particularly exciting are the possibilities in charm mixing and CP violation $[53,54,55,56]$. Charm physics also plays a crucial role in the extraction of the CKM angle $\gamma$ from $\mathrm{B}^{ \pm} \rightarrow \mathrm{D}^{0} \mathrm{~K}^{ \pm}$ decays, for 2, 3 and 4 body final states of the $\mathrm{D}$, as discussed for example in [57, 58, 59, 60, 61, 62]. CLEO-c's unique quantum correlated data provide powerful input to such measurements - see especially $[63,53,64]$ for charm mixing parameters, and $[65,66,67,68]$ for $\gamma$. The full benefit of this input, which dramatically reduces the systematic error will become apparent with LHCb's huge statistical power $[69,70,71]$. Details on these subjects can be found elsewhere in these proceedings $[2,3,4,5,1]$.

\section{Conclusion}

Hadronic charm decays address a variety of physics topic, from low energy QCD to highly sensitive probes of New Physics and crucial input to precision measurements in the B sector. In these proceedings we discussed relative and absolute branching fraction measurements in inclusive and exclusive modes, exploring flavour symmetries, strong phases, and Dalitz analyses.

Charm branching fractions shed light on low-energy QCD and its symmetries, and they provide crucial input to future measurements especially at $\mathrm{LHCb}$, which as has access to unprecedented quantities of charm and beauty, but where the measurement of absolute branching fractions is difficult. A particularly important role in this context is played by CLEO-c's very clean D $\overline{\mathrm{D}}$ samples, which allow the precise determination of absolute branching fractions, including inclusive branching fractions and and measurements with neutral decay products. The full power of these quantum-correlated $\mathrm{D} \overline{\mathrm{D}}$ events extends to many other aspects of flavour physics, especially the sensitivity to the phase difference between $\mathrm{D}$ and $\overline{\mathrm{D}}$ decay amplitudes.

A particularly powerful tool in flavour physics analyses, also through its access to phases, is the Dalitz plot analysis and its 4-body generalisation. The theoretically satisfactory, and experimentally consistent description of Dalitz plots, especially of the $\mathrm{S}$ wave contribution, remains a huge challenge. Addressing this challange becomes even more important through the enourmous data samples to be collected by LHCb and future $e^{+} e^{-}$flavour factories - requiring correspondingly high quality models to describe them in order to unlock their full potential in a variety of measurements, especially in studying $\mathrm{CP}$ violation in the charm and $\mathrm{B}$ sector, which provide highly sensitive probes for New Physics.

Two new flavour physics experiments have recently started data taking, LHCb and BES III. We are looking forward to a bright future through the combination of the unprecedented statistical power of $\mathrm{LHCb}$ in charm and B physics with the quantum correlated D $\overline{\mathrm{D}}$ pairs from CLEO-c and BES III.

\section{References}

[1] C. Thomas, D hadronic decays (related to extraction of angle $\gamma$ ), 2010, in these proceedings.

[2] N. Neri, D0 mixing, 2010, in these proceedings.

[3] M. Martinelli, Cp violation in d0 decays, 2010, in these proceedings.

[4] G. Perez, D physics: Sm and new physics potential, 2010, in these proceedings. 
[5] A. Zupanc, D(s)+ decays and their cpv, 2010, in these proceedings.

[6] CLEO, H. Mendez et al., Phys. Rev. D81, 052013 (2010), 0906.3198.

[7] B. Bhattacharya and J. L. Rosner, Phys. Rev. D81, 014026 (2010), 0911.2812.

[8] Particle Data Group, C. Amsler et al., Phys. Lett. B667, 1 (2008).

[9] L.-L. Chau, Phys. Rept. 95, 1 (1983).

[10] L. L. Chau and H. Y. Cheng, Phys. Rev. Lett. 56, 1655 (1986).

[11] M. Gronau, O. F. Hernandez, D. London, and J. L. Rosner, Phys. Rev. D50, 4529 (1994), hep-ph/9404283.

[12] J. L. Rosner, Phys. Rev. D60, 114026 (1999), hep-ph/9905366.

[13] A. Ryd and A. A. Petrov, (2009), 0910.1265.

[14] I. I. Bigi and H. Yamamoto, Physics Letters B 349, 363 (1995).

[15] R. Fleischer, Phys. Lett. B459, 306 (1999), hep-ph/9903456.

[16] R. Fleischer, Eur. Phys. J. C16, 87 (2000), hep-ph/0001253.

[17] Q. He et al., Phys. Rev. Lett. 100, 091801 (2008).

[18] S. B. Athar et al., Phys. Rev. Lett. 100, 181802 (2008).

[19] D.-N. Gao, Phys. Lett. B645, 59 (2007), hep-ph/0610389.

[20] H. J. Lipkin, Nucl. Phys. Proc. Suppl. 115, 117 (2003), hep-ph/0210166.

[21] Belle, B. R. Ko et al., Phys. Rev. Lett. 102, 221802 (2009), 0903.5126.

[22] C.-H. Chen, H.-Y. Cheng, and Y.-K. Hsiao, Phys. Lett. B663, 326 (2008), 0803.2910.

[23] BABAR, B. Aubert et al., Phys. Rev. Lett. 100, 051802 (2008), 0704.2080.

[24] CLEO, S. Dobbs et al., Phys. Rev. D76, 112001 (2007), 0709.3783.

[25] CLEO, J. P. Alexander et al., Phys. Rev. Lett. 100, 161804 (2008), 0801.0680.

[26] Belle, K. Abe et al., (2007), hep-ex/0701053.

[27] Particle Data Group, W. M. Yao et al., J. Phys. G33, 1 (2006).

[28] S. Stone, (2006), hep-ph/0605134.

[29] S. Malvezzi, AIP Conf. Proc. 549, 569 (2002).

[30] E687, P. L. Frabetti et al., Phys. Lett. B351, 591 (1995).

[31] CLEO, S. Dobbs et al., Phys. Rev. D79, 112008 (2009), 0904.2417.

[32] M. Gronau and J. L. Rosner, Phys. Rev. D79, 074022 (2009), 0903.2287.

[33] CLEO, J. Y. Ge et al., Phys. Rev. D80, 051102 (2009), 0906.2138.

[34] R. H. Dalitz, Phil. Mag. 44, 1068 (1953).

[35] E. Byckling and K. Kajantie, Particle Kinematics (John Wiley \& Sons, New York, 1973).

[36] S. M. Flatte, Phys. Lett. B 63, 224 (1976).

[37] J. Blatt and V. Weisskopf, Theoretical Nuclear Physics (John Wiley \& Sons, New York, 1952). 
[38] E. P. Wigner, Phys. Rev. 70, 15 (1946).

[39] I. J. R. Aitchison, Nucl. Phys. A189, 417 (1972).

[40] E791, E. M. Aitala et al., Phys. Rev. Lett. 86, 770 (2001), hep-ex/0007028.

[41] FOCUS, J. M. Link et al., Phys. Lett. B 585, 200 (2004), hep-ex/0312040.

[42] CLEO, G. Bonvicini et al., Phys. Rev. D 76, 012001 (2007), 0704.3954 [hep-ex].

[43] J. Schechter, Int. J. Mod. Phys. A 20, 6149 (2005), hep-ph/0508062, and references in [42].

[44] N. N. Achasov and G. N. Shestakov, Phys. Rev. D 67, 114018 (2003), hep-ph/0302220, and further references in [42].

[45] Particle Data Group, W. M. Yao et al., J. Phys. G33, 1 (2006).

[46] E791, E. M. Aitala et al., Phys. Rev. Lett. 89, 121801 (2002), hep-ex/0204018.

[47] E791, E. M. Aitala et al., Phys. Rev. D 73, 032004 (2006), hep-ex/0507099, [Erratum-ibid. D 74 (2006), 059901].

[48] FOCUS, J. M. Link et al., Phys. Lett. B 653, 1 (2007), 0705.2248 [hep-ex], doi:10.1016/j.physletb.2003.10.071, and CLEO-c [42].

[49] D. Aston et al., Nucl. Phys. B 296, 493 (1988).

[50] J. Link et al., Physics Letters B 681, 14 (2009).

[51] CLEO, G. Bonvicini et al., Phys. Rev. D 78, 052001 (2008), 0802.4214 [hep-ex].

[52] FOCUS, J. M. Link et al., Phys. Rev. D 75, 052003 (2007), hep-ex/0701001.

[53] CLEO, D. M. Asner et al., Phys. Rev. D72, 012001 (2005), hep-ex/0503045.

[54] BELLE, K. Abe et al., Phys. Rev. Lett. 99, 131803 (2007), 0704.1000.

[55] BABAR Collaboration, P. del Amo Sanchez et al., Phys. Rev. Lett. 105, 081803 (2010).

[56] Belle Collaboration, L. M. Zhang et al., Phys. Rev. Lett. 99, 131803 (2007).

[57] M. Gronau and D. Wyler, Phys. Lett. B 265, 172 (1991).

[58] M. Gronau and D. London, Phys. Lett. B 253, 483 (1991).

[59] D. Atwood, I. Dunietz, and A. Soni, Phys. Rev. Lett. 78, 3257 (1997), hep-ph/9612433.

[60] A. Giri, Y. Grossman, A. Soffer, and J. Zupan, Phys. Rev. D 68, 054018 (2003).

[61] A. Bondar and A. Poluektov, (2007), hep-ph/0703267.

[62] J. Rademacker and G. Wilkinson, Phys. Lett. B 647, 400 (2007), hep-ph/0611272.

[63] CLEO, D. M. Asner et al., Phys. Rev. D78, 012001 (2008), 0802.2268.

[64] A. Bondar, A. Poluektov, and V. Vorobiev, Phys. Rev. D 82, 034033 (2010).

[65] D. Atwood and A. Soni, Phys. Rev. D 68, 033003 (2003), hep-ph/0304085.

[66] CLEO, R. A. Briere et al., Phys. Rev. D80, 032002 (2009), 0903.1681.

[67] CLEO, N. Lowrey et al., Phys. Rev. D80, 031105 (2009), 0903.4853.

[68] CLEO Collaboration, J. Libby et al., Phys. Rev. D82, 112006 (2010), 1010.2817.

[69] J. Libby, A. Powell, G. Wilkinson, and J. Rademacker, CERN-LHCB-2007-098.

[70] K. Akiba et al., Report No. CERN-LHCB-2008-031.

[71] The LHCb Collaboration, (2009), 0912.4179. 